In Intl. Trans. in Operational Research, vol. 9, no. 1, January 2002, pp. 97-112.

\title{
Generalized Loss Models and Queueing-Loss Networks
}

\author{
Hisashi Kobayashi \\ Dept. of Electrical Engineering \\ School of Engineering and Applied Science \\ Princeton University \\ Princeton, NJ 08544, U.S.A.
}

\author{
Brian L. Mark \\ Dept. of Electrical and Computer Eng. \\ School of Information Technology and Eng. \\ George Mason University \\ Fairfax, VA 22030, U.S.A.
}

\begin{abstract}
The classical Erlang and Engset loss models have been used extensively in the traffic engineering of traditional telephone exchanges. More recently, these models have been generalized to the so-called loss networks, which provide models for resource sharing in multiservice telecommunication networks. In this paper, we introduce a new generalized class of models, queueing-loss networks, which captures both queueing and loss aspects of a system. The queueing-loss network model is a natural extensions of queueing networks and loss networks which have the product-form solution. We discuss applications of the model and analyze a particular example of a simple queueing-loss network.
\end{abstract}

Key words: loss models, loss networks, queueing models, queueing networks, product-form

\section{Introduction}

Recently, there has been an increasing interest in generalizations of the loss models originally studied by Erlang and Engset in the context of telephone exchanges (see e.g., (Syski, 1986)). Loss networks provide models for studying the blocking behavior of connection-oriented services in circuit-switched networks, ATM networks, optical networks and wireless networks. As we discuss in this paper, loss networks have much in common with the traditional queueing network models. The earliest work on queueing networks with product form goes back to J.R. Jackson's original paper (1963). Theory for queueing network models has advanced considerably over the past several decades (Baskett et al, 1975), (Kelly, 1979), (Reiser \& Kobayashi, 1975) and has been widely applied to the performance analysis of computing systems and packet-switched networks (Kobayashi, 1978).

This paper introduces a new class of models, queueing-loss networks, which are natural generalizations of queueing networks and loss networks. We give a brief development of loss networks by systematically generalizing the classical loss models using notions from the theory of queueing networks. This development culminates in the introduction of queueing-loss networks and a discussion of their properties. Next, possible applications of the model are discussed and a particular example of a simple queueing-network model is analyzed. Finally, the paper concludes with a discussion of directions for further research on queueing-loss network models. 


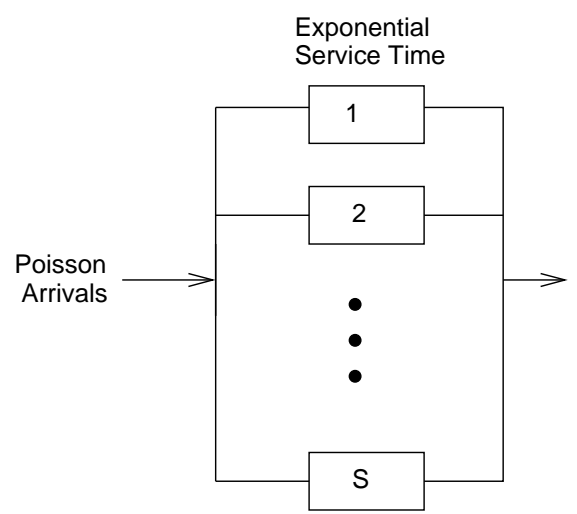

Figure 1: Erlang Loss Model

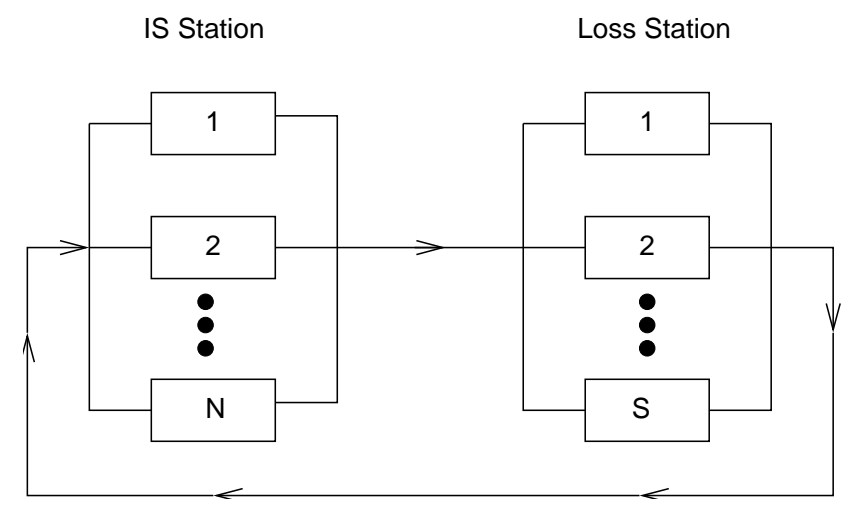

Figure 2: Engset Loss Model

\section{Generalized Loss Models}

We use the general term station, to denote an entity which provides service to arriving calls or customers. A station consists of a number of servers or lines and possibly a waiting room or buffer. A loss station is one that has a finite number of servers and no waiting room. An arriving call either begins service immediately or is rejected due to the lack of a sufficient number of available servers. By contrast, a queueing station, as considered in this paper, has a sufficiently large waiting room such that no call is rejected.

The original loss model studied by Erlang is equivalent to an $M / M / S(0)$ queue ${ }^{1}$ (see Figure 1); i.e., a loss station with $S$ servers where arriving calls form a Poisson process with rate $\lambda$ and each call occupies a server for an exponentially distributed holding time with mean $1 / \mu$. The stationary distribution of the number of busy servers is given by

$$
P(n)=\frac{1}{G(S)} \frac{a^{n}}{n !}, \quad 0 \leq n \leq S,
$$

where $a=\lambda / \mu$ is the offered load and $G(S)$ is a normalization constant given by

$$
G(S)=\sum_{n=0}^{S} \frac{a^{n}}{n !}
$$

\footnotetext{
${ }^{1}$ Often the notation $M / M / S / S$ is used in the queueing theory literature, where the second $S$ signifies the maximum number of calls that can be accommodated in the system.
} 
As $S \rightarrow \infty, G(S) \rightarrow e^{a}$, hence $P(n) \rightarrow a^{n} e^{-a} / n$ !, which is the stationary distribution of an infinite-server (IS) station or $M / M / \infty$ queue (see e.g., (Kobayashi, 1978)). Therefore, the distribution (1) is a truncated Poisson distribution. The probability that all servers are found busy in the steady state is given by the celebrated Erlang loss formula:

$$
B(S) \stackrel{\text { def }}{=} P(S)=\frac{a^{S}}{S !}\left[\sum_{i=0}^{S} \frac{a^{i}}{i !}\right]^{-1} .
$$

The Erlang loss formula can be expressed in terms of the normalization constant as follows:

$$
B(S)=1-\frac{G(S-1)}{G(S)} .
$$

The above probability $B(S)$ is often referred to as the time congestion, since this represents the proportion of time that all the servers are busy. The call congestion or call loss probability $L(S)$ is defined as the probability that a newly arriving call finds all servers occupied, and hence is lost or blocked, i.e., leaves the system without being served. When the arrival process is Poisson, as in the Erlang loss model, the call congestion and the time congestion can be seen to be equivalent, via the so-called PASTA (Poisson Arrivals See Time Averages) property (Wolff, 1989).

If we replace the Poisson arrival (i.e., an infinite source model) in the Erlang loss model by a finite number $N$ of sources $(N>S)$, then we obtain what is often termed the Engset loss model (see Figure 2), which we denote as an $M(N) / M / S(0)$ queue $^{2}$. Each source generates a call with an exponentially distributed inter-generation time with mean $1 / \nu$ and then places the call at the loss station, where it either acquires a server for an exponentially distributed holding time or is blocked. Both completed and lost calls alike return to the sources and a new cycle begins. For this model, $n(t)$, the number of calls in progress at time $t$, will have, in the steady state, the following truncated binomial distribution:

$$
P(n, N)=\frac{1}{G(S, N)}\left(\begin{array}{l}
N \\
n
\end{array}\right) b^{n}, \quad 0 \leq n \leq S,
$$

where $b=\nu / \mu$ and the normalization constant $G(S, N)$ is given by

$$
G(S, N)=\sum_{n=0}^{S}\left(\begin{array}{l}
N \\
n
\end{array}\right) b^{n} .
$$

The time congestion $B(S, N)$ is given by

$$
B(S, N) \stackrel{\text { def }}{=} P(S, N)=1-\frac{G(S-1, N)}{G(S, N)} .
$$

Because the arrival is not Poisson in the finite source model, the call congestion $L(S, N)$ is no longer the same as $B(S, N)$, but we find the following simple relation:

$$
L(S, N)=1-\frac{G(S-1, N-1)}{G(S, N-1)}=B(S, N-1) .
$$

\footnotetext{
${ }^{2}$ In the literature it is often referred to as $M / M / S / N / S$, where the last two symbols represent the number of sources, and the number of customers that can be accommodated in this service station.
} 
More generally, the distribution of number of calls in service seen by an arriving call is the time average distribution that would be observed if the number of sources were reduced by one. This is analogous to the result that holds in an $M(N) / M / 1$ queue or a machine servicing model (Kobayashi, 1978).

We now define a generalized Erlang loss model as follows:

1. Multi-class sources: We introduce a set, $\mathcal{C}$, of call classes. The arrival pattern of class $c$ calls is a Poisson process with rate $\lambda_{c}$. We denote by $n_{c}(t)$ the number of class $c$ calls in progress at time $t$.

2. Simultaneous acquisition of multiple servers: A class $c$ call requires to hold $A_{c}$ servers simultaneously. If the total number of servers or lines is $S$, then the following constraint must be met:

$$
\sum_{c \in \mathcal{C}} A_{c} n_{c}(t) \leq S
$$

3. Generally distributed holding time: The call holding time distribution is a general distribution $G_{c}(t)$ with mean $1 / \mu_{c}$ :

$$
\int_{0}^{\infty}\left(1-G_{c}(t)\right) d t=\frac{1}{\mu_{c}}
$$

Let the state process of this generalized loss station be denoted by $\mathbf{n}(t)=\left(n_{c}(t): c \in \mathcal{C}\right)$. Let $P(\mathbf{n})$ denote the equilibrium state distribution when there are $S$ servers. The set of feasible states is

$$
\mathcal{F}(S)=\left\{\mathbf{n} \geq \mathbf{0}: \sum_{c \in \mathcal{C}} A_{c} n_{c} \leq S\right\} .
$$

The departure process from the station includes both calls that have successfully completed service and those which are rejected. The generalized Erlang station shares many of the properties associated with stations in queueing networks.

A queueing station is said to be quasi-reversible if its state process $\mathbf{n}(t)$ is a stationary Markov process with the property that the state at an arbitrary time $t_{0}$ is independent of:

(i) the arrival times of class $c$ calls, $c \in \mathcal{C}$, after time $t_{0}$; and

(ii) the departure times of class $c$ calls, $c \in \mathcal{C}$, prior to time $t_{0}$.

The property of quasi-reversibility was introduced by Kelly (1979) to characterize a wide class of queueing stations which, together with certain rules governing call routing, give rise to productform queueing networks. We extend this definition to loss stations by assuming the convention that the departure process includes both calls that successfully complete service and those which are blocked and do not receive service. A closely related property is reversibility. A stochastic process $n(t)$ is reversible if it is statistically identical with the time reversed process $n_{R}(t)=n(\tau-t)$ for any $\tau$. For a stationary Markov process, reversibility holds if and only if 
its stationary distribution satisfies the detailed balance equations (Baskett et al, 1975), (Kelly, 1979).

The following important theorem is proved in (Kobayashi \& Mark, 1994):

Theorem 2.1 The generalized Erlang station is quasi-reversible and its state-process $\mathbf{n}(t)$ is a reversible Markov process with stationary distribution given by

$$
P(\mathbf{n} \mid S)=\frac{1}{G(S)} \prod_{c \in \mathcal{C}} \frac{a_{c}{ }^{n_{c}}}{n_{c} !}, \quad \mathbf{n} \in \mathcal{F}(S)
$$

where $a_{c}=\lambda_{c} / \mu_{c}$ and $G(S)$ is the normalization constant defined by

$$
G(S)=\sum_{\mathbf{n} \in \mathcal{F}(S)} \prod_{c \in \mathcal{C}} \frac{a_{c}^{n_{c}}}{n_{c} !}
$$

This result can be easily extended to the loss station model in which the calls are generated from a finite number of sources of multiple classes. We define a generalized Engset loss station as follows:

1. Multi-class sources: Let $N_{c}$ be the number of sources for class $c$ calls, $c \in \mathcal{C}$, and let $\mathbf{N}$ be the vector $\left\{N_{c}, c \in \mathcal{C}\right\}$. We denote by $n_{c}(t)$ the number of class $c$ calls in progress at time t. Then, clearly

$$
n_{c}(t) \leq N_{c}, \quad c \in \mathcal{C}
$$

The inter-generation time at a class- $c$ source is characterized by a general distribution $F_{c}(t)$ with mean $1 / \nu_{c}$ :

$$
\int_{0}^{\infty}\left(1-F_{c}(t)\right) d t=\frac{1}{\nu_{c}} .
$$

2. Simultaneous acquisition of multiple servers: As in the generalized Erlang loss model.

3. Generally distributed holding time: As in the generalized Erlang loss model.

The set of feasible states is now given by

$$
\mathcal{F}(S, \mathbf{N})=\left\{\mathbf{n} \geq \mathbf{0}: \sum_{c \in \mathcal{C}} A_{c} n_{c} \leq S ; \quad n_{c} \leq N_{c}, c \in \mathcal{C}\right\}
$$

The following theorem (Kobayashi \& Mark, 1994) is a generalization of a result first reported by Cohen (1957).

Theorem 2.2 For the generalized Engset loss system, $\mathbf{n}(t)$ is a reversible Markov process with stationary distribution:

$$
P(\mathbf{n} \mid S, \mathbf{N})=\frac{1}{G(S, \mathbf{N})} \prod_{c \in \mathcal{C}}\left(\begin{array}{l}
N_{c} \\
n_{c}
\end{array}\right) b_{c}{ }^{n_{c}}, \quad \mathbf{n} \in \mathcal{F}(S, \mathbf{N})
$$

where $b_{c}=\nu_{c} / \mu_{c}$, and the normalization constant $G(S, \mathbf{N})$ is given by

$$
G(S, \mathbf{N})=\sum_{\mathbf{n} \in \mathcal{F}(S, \mathbf{N})} \prod_{c \in \mathcal{C}}\left(\begin{array}{l}
N_{c} \\
n_{c}
\end{array}\right) b_{c}{ }^{n_{c}}
$$




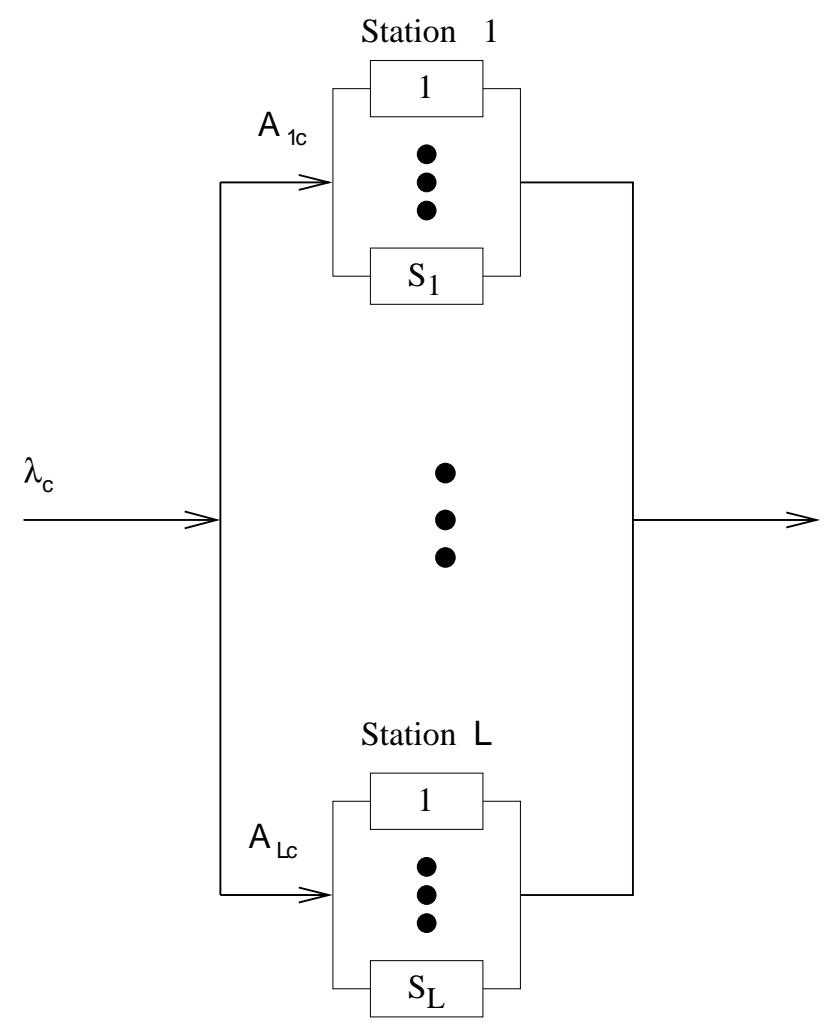

Figure 3: Generalized Loss Station

\section{Loss Networks}

One can further extend the above generalized loss station (GLS) models by introducing multiple server types. In the generalized Erlang and Engset models, we extend the second property as follows:

2'. Simultaneous acquisition of multiple servers of different types: Let $\mathcal{L}$ denote a set of server types. There are $S_{l}$ servers of type $l \in \mathcal{L}$. A class $c$ call requires to hold $A_{l c}$ servers of type $l$ simultaneously. For each server type $l \in \mathcal{L}$, the following constraint must be met:

$$
\sum_{c \in \mathcal{C}} A_{l c} n_{c}(t) \leq S_{l}
$$

The results of Theorems 3.1 and 3.2 can be generalized straightforwardly to accommodate the concept of server types. Figure 3 shows a generalized loss station in which each call can simultaneously acquire multiple servers from among several server types.

We define the properties of a loss network as follows:

1. Let $\mathcal{L}$ denote the set of links in the loss network. A link $\ell \in \mathcal{L}$ contains $S_{\ell}$ channels.

2. A call class $c \in \mathcal{C}$ is defined as a pair $(r, \tau)$, where $r \in \mathcal{R}$ is a route or path of the call in the loss network, and $\tau \in \mathcal{T}$ is the type of the call. Thus, the set $\mathcal{C}=\mathcal{R} \times \mathcal{T}{ }^{3}$

\footnotetext{
${ }^{3}$ In the loss station models discussed in the preceding section, the class $\mathcal{C}$ and the type $\mathcal{T}$ are equivalent. In the loss network, for a given source-destination pair, different types of call may take different routes.
} 


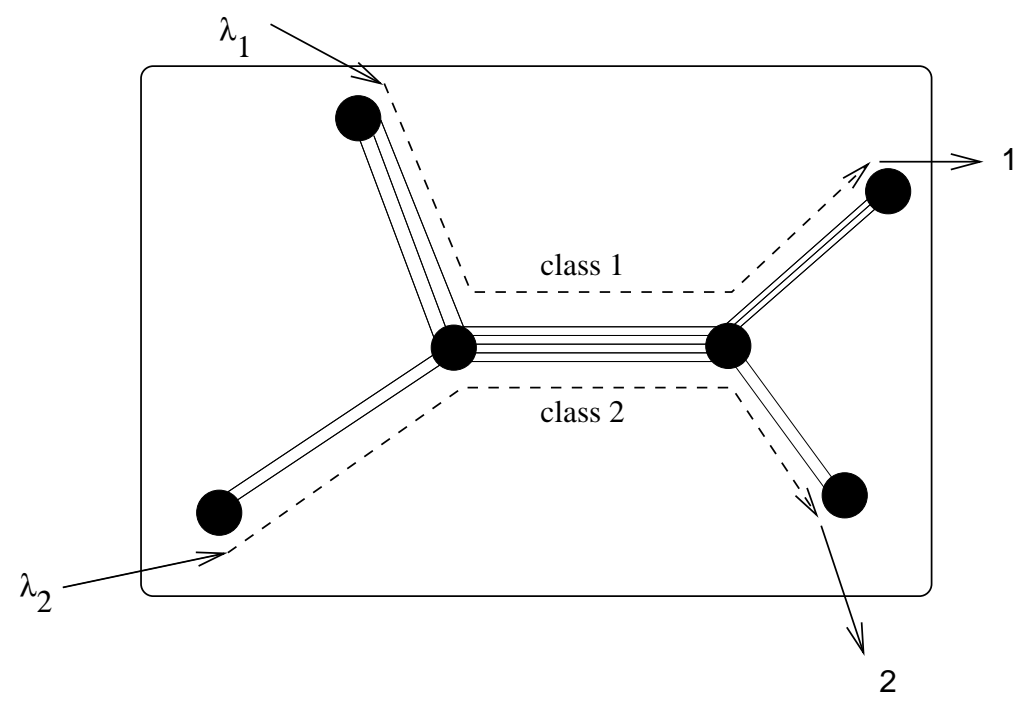

Figure 4: Open Loss Network

3. A class $c$ call seeks to simultaneously acquire $A_{\ell c}$ channels of link $\ell$ for each link $\ell \in \mathcal{L}$.

4. The holding time of a class $c$ call is a general distribution $G_{c}(t)$ with mean $1 / \mu_{c}$.

The loss network can be seen to be equivalent to a generalized loss station (GLS) with multiple server types, where each link in the loss network corresponds to a server type in the GLS. The loss network provides a general model for a circuit-switched network that carries multi-rate traffic (i.e., different values of $A_{\ell c}$ for different $c$ ) among different types $\tau$ of calls (Kelly, 1991), (Kobayashi \& Mark, 1997). The model is equally applicable to bidirectional flows. All that is required is to assign different class parameters to traffic in the reverse directions. The reverse traffic for a given pair of nodes may have different bandwidth requirements (i.e., $A_{\ell c}$ different from that for the forward direction). Similarly, the route used for the traffic in the reverse direction need not be the reverse path of the forward path.

By specifying the arrival pattern of a loss network as a multi-class Poisson process as in property 1 of the generalized Erlang model in section 2, we obtain an open loss network (OLN) (see Figure 4). The OLN is equivalent to a generalized Erlang loss station with simultaneous acquisition among multiple server types. If we replace the multi-class Poisson process of the OLN by a multi-class finite source model as in property 1 of the generalized Engset model, we obtain a closed loss network (CLN). The CLN is equivalent to a generalized Engset loss station with multiple server types.

In the open loss network, the Poisson stream of class $c$ arrivals is analogous to an open subchain in a queueing network (Baskett et al, 1975), (Reiser \& Kobayashi, 1975). Hence, in the OLN, each class $c$ is said to be open. Similarly, dual to the concept of a closed subchain in a queueing network, we can define a closed class $c$ in a loss network by replacing the Poisson stream of class $c$ call arrivals by a finite source model of population $N_{c}$. The closed loss network is then a loss network wherein all the classes are closed. A mixed loss network (MLN), as shown 


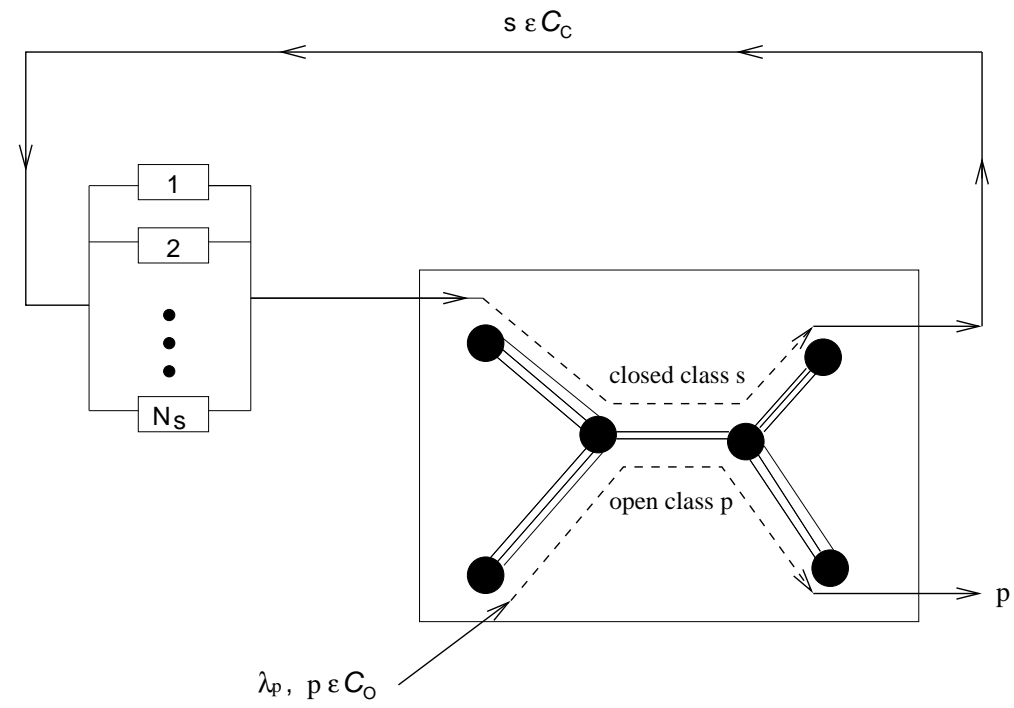

Figure 5: Mixed Loss Network

in Figure 5, may have both kinds of classes. The MLN further generalizes the generalized Erlang and Engset stations of the previous section. Open, closed and mixed loss networks are analogous to open, closed and mixed queueing networks (Baskett et al, 1975), respectively.

Denote the state process of a mixed loss network by $\mathbf{n}(t)=\left[\mathbf{n}_{O}(t), \mathbf{n}_{C}(t)\right]$, with $\mathbf{n}_{O}(t)=$ $\left(n_{c}(t): c \in \mathcal{C}_{O}\right)$ and $\mathbf{n}_{C}(t)=\left(n_{c}(t): c \in \mathcal{C}_{C}\right)$. We have the following result for the MLN:

Theorem 3.1 The state process of the mixed loss network is a reversible Markov process with equilibrium distribution given by

$$
P(\mathbf{n} \mid \mathbf{S}, \mathbf{N})=\frac{1}{G(\mathbf{S}, \mathbf{N})} P_{O}\left(\mathbf{n}_{O}\right) P_{C}\left(\mathbf{n}_{C} \mid \mathbf{N}\right), \quad \mathbf{n} \in \mathcal{F}(\mathbf{S}, \mathbf{N})
$$

where

$$
P_{O}\left(\mathbf{n}_{O}\right)=\prod_{c \in \mathcal{C}_{O}} \frac{a_{c}^{n_{c}}}{n_{c} !}, \quad P_{C}\left(\mathbf{n}_{C} \mid \mathbf{N}\right)=\prod_{c \in \mathcal{C}_{C}}\left(\begin{array}{c}
N_{c} \\
n_{c}
\end{array}\right) b_{c}{ }^{n_{c}}
$$

with $a_{c}=\lambda_{c} / \mu_{c} \quad\left(c \in \mathcal{C}_{O}\right), b_{c}=\nu_{c} / \mu_{c}, \quad\left(c \in \mathcal{C}_{C}\right)$, and

$$
\mathcal{F}(\mathbf{S}, \mathbf{N})=\left\{\mathbf{n} \geq \mathbf{0} ; \quad \sum_{c \in \mathcal{C}} A_{\ell c} n_{c} \leq S_{\ell}, \ell \in \mathcal{L} ; \quad n_{c} \leq N_{c}, c \in \mathcal{C}_{C}\right\}
$$

and

$$
G(\mathbf{S}, \mathbf{N})=\sum_{\mathbf{n} \in \mathcal{F}(\mathbf{S}, \mathbf{N})} P_{O}\left(\mathbf{n}_{O}\right) P_{C}\left(\mathbf{n}_{C}\right)
$$

From the stationary distribution of the mixed loss network obtained above, we can express the time congestion and call congestion for the class $c$ in terms of the normalization constant $G(\mathbf{S}, \mathbf{N})$ as follows: 


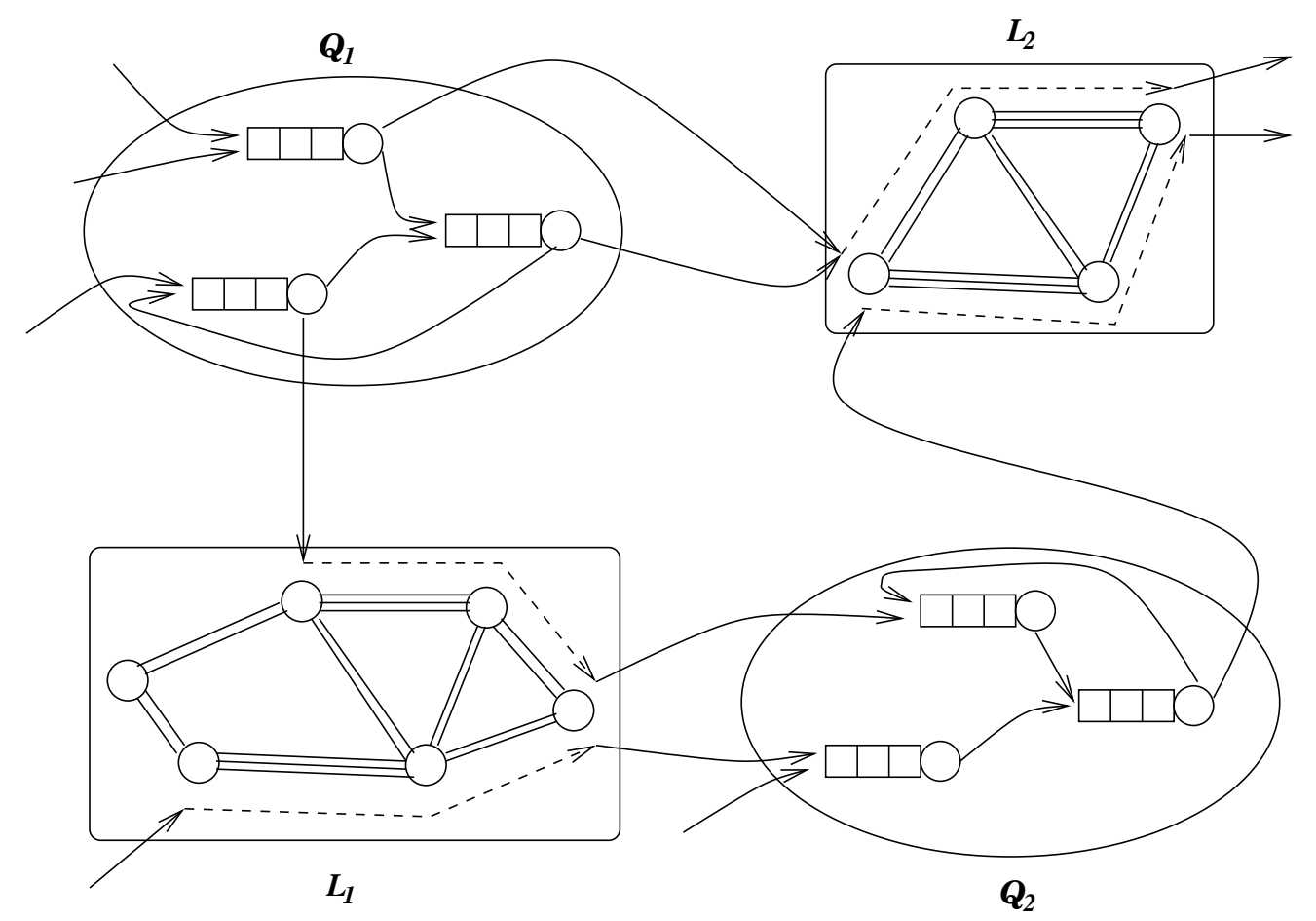

Figure 6: Queueing-Loss Network

1. For a class $c$ call in an open route $c \in \mathcal{C}_{O}$

$$
\begin{aligned}
& B_{c}(\mathbf{S}, \mathbf{N})=1-\frac{G\left(\mathbf{S}-\mathbf{A}_{c}, \mathbf{N}\right)}{G(\mathbf{S}, \mathbf{N})} \\
& L_{c}(\mathbf{S}, \mathbf{N})=B_{c}(\mathbf{S}, \mathbf{N})
\end{aligned}
$$

where $\mathbf{A}_{c}$ is the $c$-th column of the matrix $\mathbf{A}=\left[A_{\ell c}\right]$. The last equation is due to the PASTA property referred to earlier.

2. For a class $c$ call in a closed route $c \in \mathcal{C}_{C}$

$$
\begin{aligned}
& B_{c}(\mathbf{S}, \mathbf{N})=1-\frac{G\left(\mathbf{S}-\mathbf{A}_{c}, \mathbf{N}\right)}{G(\mathbf{S}, \mathbf{N})} \\
& L_{c}(\mathbf{S}, \mathbf{N})=B_{c}\left(\mathbf{S}, \mathbf{N}-\mathbf{1}_{c}\right),
\end{aligned}
$$

where $\mathbf{1}_{c}$ denotes the unit vector, whose $c$-th component is unity.

The above formulas for the time and call congestions are generalizations of the formulas obtained for the Erlang and Engset models. For numerical methods (exact, approximate and asymptotic) to compute the normalization constants $G(\mathbf{S}, \mathbf{N})$ for different values of $\mathbf{S}$ and $\mathbf{N}$, the reader is referred to (Kobayashi \& Mark, 1997) and references cited therein.

\section{Queueing-Loss Networks}

Thus far, we have arrived at the mixed loss network by generalizing the classical Erlang and Engset loss models. We now carry the generalization further by introducing the concept of 
a queueing-loss network (QLN). A queueing-loss network (see Figure 6) consists of a set of queueing subnetworks $\left\{\mathcal{Q}_{j} ; j \in \mathcal{J}\right\}$ and a set of loss subnetworks $\left\{\mathcal{L}_{k} ; k \in \mathcal{K}\right\}$. Calls are routed within each queueing subnetwork and loss subnetwork component as well as between queueing and loss network components. The call routing behavior can be governed by a Markov chain of arbitrary order (Kobayashi, 1978).

Each queueing subnetwork, $\mathcal{Q}_{j}$, consists of a network of quasi-reversible queueing stations. Hence, if $\mathbf{n}_{\mathcal{Q}_{j}}$ denotes the population vector in the queueing subnetwork $\mathcal{Q}_{j}$, its stationary state distribution $P_{\mathcal{Q}_{j}}\left(\mathbf{n}_{\mathcal{Q}_{j}}\right)$ has the product form. Furthermore, the queueing network itself is quasi-reversible (Kelly, 1979).

In general, each loss subnetwork, $\mathcal{L}_{k}$, can be a mixed loss network (MLN). The loss network component of the MLN can be replaced by an equivalent Generalized Loss Station (GLS) with simultaneous server acquisition. Each closed class in the MLN, representing a finite source population, can be decomposed as infinite server (IS) station placed in tandem with the GLS (see Figure 5). Let $\mathbf{n}_{\mathcal{L}_{k}}$ denote the population vector for the MLN. From Theorem 3.1, the state distribution $P_{\mathcal{L}_{k}}\left(\mathbf{n}_{\mathcal{L}_{k}}\right)$ has the product form. In the decomposed representation of the MLN, each IS component is quasi-reversible, and by Theorem 2.1, the GLS component is also quasi-reversible.

Hence, the queueing-loss network can be decomposed into a set of quasi-reversible components. The routing of calls between these components can be characterized by a Markov chain of arbitrary order (Kobayashi, 1978). By combining these observations we now see that the QLN is a generalized queueing network. The only difference between this queueing network and those studied previously (Baskett et al, 1975), (Kelly, 1979), (Reiser \& Kobayashi, 1975) is that it contains GLSs as its components. We have already established the fact that a GLS is a generalized version of an IS station, and is a quasi-reversible station. Therefore, we can conclude that the QLN has a product-form solution. We state this general result for queueing-loss networks in the following theorem:

Theorem 4.1 Consider a queueing-loss network ( $Q L N)$ that contains a set of queueing subnetworks $\left\{\mathcal{Q}_{j} ; j \in \mathcal{J}\right\}$ and a set of loss subnetworks $\left\{\mathcal{L}_{k} ; k \in \mathcal{K}\right\}$. Let $\mathbf{n}_{\mathcal{Q}_{j}}$ and $\mathbf{n}_{\mathcal{L}_{k}}$ represent the population vectors in these subnetworks. The joint stationary distribution of the state process $\mathbf{n}(t)$ of the $Q L N$ takes the form:

$$
P(\mathbf{n})=\frac{1}{G(\mathbf{S}, \mathbf{N})} \prod_{j \in \mathcal{J}} P_{\mathcal{Q}_{j}}\left(\mathbf{n}_{\mathcal{Q}_{j}}\right) \prod_{k \in \mathcal{K}} P_{\mathcal{L}_{k}}\left(\mathbf{n}_{\mathcal{L}_{k}}\right)
$$

where $P_{\mathcal{Q}_{j}}(\cdot)$ and $P_{\mathcal{L}_{k}}(\cdot)$ themselves have product forms and are proportional to the marginal distributions of the subnetworks $\mathcal{Q}_{j}$ and $\mathcal{L}_{k}, j \in \mathcal{J}, k \in \mathcal{K}$. The normalization constant $G(\mathbf{S}, \mathbf{N})$ and the feasible state set $\mathcal{F}(\mathbf{S}, \mathbf{N})$ are defined over the capacity vector $\mathbf{S}$ of loss stations, and the finite source vector $\mathbf{N}$ in the network. These vectors correspond to Cartesian products of the corresponding vectors of the subnetworks. 


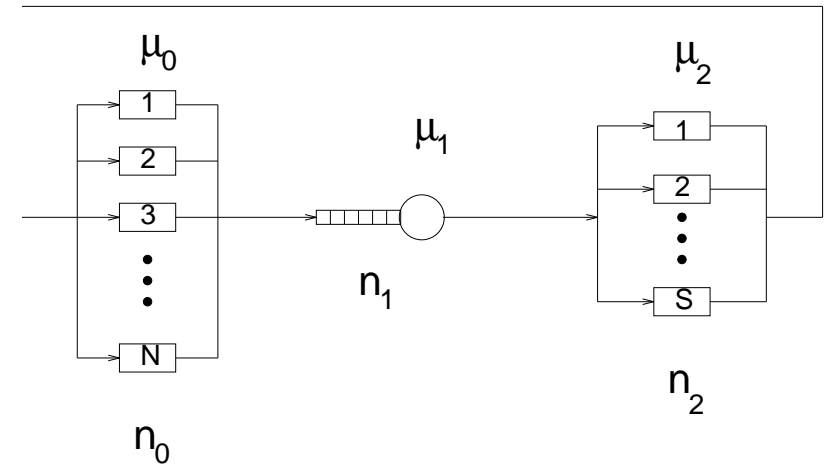

Figure 7: Example of a Queueing-Loss Network

\section{$5 \quad$ Example Queueing-Loss Network}

A useful application of the queueing-loss network model may be found, for example, in a circuitswitched network in which call connection requests are served by either a centralized facility or distributed centers. Arriving calls may have to queue for the call-connection service if many such requests are already placed on the call-connection server. The call-connection server performs the function of admission control; i.e., it decides whether a new call can be accepted or not, based on the bandwidth resources requested by the call and the available resources of the network. In this application, the call-connection server is modeled by a queueing station, whereas the circuit-switched network itself is modeled by a loss network. The overall system is thus modeled by a queueing-loss network.

Consider the simple example of a QLN shown in Figure 7. We label the three stations as stations 0,1 , and 2 , respectively. Station 0 is an IS station, representing a finite source of population $N$. The inter-generation time of calls from each source is given by a general distribution. Station 1 is a single server queue representing, for example, a call-connection server. Station 2 is a loss station with $S$ servers, and the call holding time can have a general distribution. We assume that $N>S$; otherwise, a call loss would not occur at this station. For the application discussed above, station 2 could be replaced by a more general loss network, representing, for example, a circuit-switched network.

As the results in the previous sections suggest, we can allow multiple classes of sources at station 0 , and multiple types of servers at station 2. If station 1's queue discipline is FCFS (first-come, first-served) or any type of work-conserving queue discipline, then the service times at this station must be drawn from the exponential service time that is common to all classes of customers (see e.g, (Kobayashi, 1978)). If the queue discipline of station 1 is either LCFS-PR (last come, first-served with preemptive resume) or processor sharing (PS), then we can allow multiple classes for the service time and the distribution functions can be general, as long as their means are finite. We should also note that the processing rate of each station can be queue-dependent, i.e., the completion rate $\mu_{i}\left(n_{i}\right)$ of each server at station $i$ can be an arbitrary function of its local queue size $n_{i}, i=0,1,2$. For the IS station and the loss station, we allow 
the dependency $\mu_{c}\left(n_{i c}\right)$ for different classes $c \in \mathcal{C}, \quad i=0,2$. The same generality applies to a queueing station as well, if it adopts either LCFS-PR or PS.

For the sake of illustrative simplicity, we assume only one class of sources and a single type of servers at the loss station, i.e., station 2. Further, we assume that the service rates are queue independent. Thus, the inter-generation time of each source at station 0 has mean $1 / \mu_{0}$, the service time at station 1 is exponentially distributed with mean $1 / \mu_{1}$, and the service time at station 2 has mean $1 / \mu_{2}$. Using Theorem 4.1 , we can write the stationary distribution of the queueing-loss network as:

$$
\begin{aligned}
P\left(n_{0}, n_{1}, n_{2}\right) & \propto \frac{1}{n_{0} !}\left(\frac{\lambda}{\mu_{0}}\right)^{n_{0}}\left(\frac{\lambda}{\mu_{1}}\right)^{n_{1}} \frac{1}{n_{2} !}\left(\frac{\lambda}{\mu_{2}}\right)^{n_{2}} \\
& \propto \frac{1}{n_{0} !}\left(\frac{1}{\mu_{0}}\right)^{n_{0}}\left(\frac{1}{\mu_{1}}\right)^{n_{1}} \frac{1}{n_{2} !}\left(\frac{1}{\mu_{2}}\right)^{n_{2}}
\end{aligned}
$$

for $\left(n_{0}, n_{1}, n_{2}\right)$ in the feasible set

$$
\mathcal{F}(S, N)=\left\{\left(n_{0}, n_{1}, n_{2}\right): n_{0}+n_{1}+n_{2}=N ; n_{0}, n_{1} \geq 0 ; 0 \leq n_{2} \leq S\right\}
$$

Here, $\lambda$ is the rate of traffic through this closed route, but this unknown parameter can be absorbed into the normalization constant. Hence, we can write

$$
P\left(n_{0}, n_{1}, n_{2}\right)=\frac{1}{G(S, N)} \frac{1}{n_{0} !}\left(\frac{1}{\mu_{0}}\right)^{n_{0}}\left(\frac{1}{\mu_{1}}\right)^{n_{1}} \frac{1}{n_{2} !}\left(\frac{\mu_{0}}{\mu_{2}}\right)^{n_{2}}
$$

where

$$
\begin{aligned}
G(S, N) & =\sum_{n_{0}+n_{1}+n_{2}=N, 0 \leq n_{2} \leq S} \frac{1}{n_{0} !}\left(\frac{1}{\mu_{0}}\right)^{n_{0}}\left(\frac{1}{\mu_{1}}\right)^{n_{1}} \frac{1}{n_{2} !}\left(\frac{1}{\mu_{2}}\right)^{n_{2}} \\
& =\frac{1}{\mu_{1}^{N}} \sum_{n_{2}=0}^{S} \sum_{n_{0}=0}^{N-n_{2}} \frac{1}{n_{0} !}\left(\frac{1}{\mu_{0}}\right)^{n_{0}} \frac{1}{n_{2} !}\left(\frac{\mu_{0}}{\mu_{2}}\right)^{n_{2}} .
\end{aligned}
$$

The time congestion and call congestion at station 2 are then obtained by

$$
\begin{aligned}
& B(S, N)=1-\frac{G(S-1, N)}{G(S, N)}, \\
& L(S, N)=B(S, N-1)=1-\frac{G(S-1, N-1)}{G(S, N-1)} .
\end{aligned}
$$

Suppose that we wish to find $\rho_{1}$, the utilization of station 1. By extending results known for closed queueing networks (see e.g., (Kobayashi, 1978)), we can write

$$
\rho_{1}=1-\frac{G^{(-1)}(S, N)}{G(S, N)}
$$

where $G^{(-1)}(S, N)$ represents the value of the normalization constant when station 1 is deleted from the system. This corresponds to the situation which would arise if we let $\mu_{1} \rightarrow \infty$ in the above queueing-loss system. In the limit as $\mu_{1} \rightarrow \infty$, only the terms corresponding to $n_{1}=0$ remain in (31) and we obtain the following expression for $G^{(-1)}(S, N)$ :

$$
G^{(-1)}(S, N)=\frac{1}{N !}\left(\frac{1}{\mu_{0}}\right)^{N} G_{L}(S, N)
$$


where

$$
G_{L}(S, N)=\sum_{n=0}^{S}\left(\begin{array}{l}
N \\
n
\end{array}\right)\left(\frac{\mu_{0}}{\mu_{2}}\right)^{n}
$$

is the normalization constant of the Engset loss station resulting from deleting station 1 from the queueing-loss network. If station 1 has a constant rate, as is the present case, we can use the following alternative formula (see e.g., (Kobayashi, 1979, p. 172)):

$$
\rho_{1}=\frac{1}{\mu_{1}} \frac{G(S, N-1)}{G(S, N)}
$$

It is not difficult to confirm that the above two formulas for the server utilization $\rho_{1}$ are indeed equivalent.

To study the effect of the queueing station 1 on the system capacity of loss station 2 , one can express the marginal distribution of station 2 as a function of $\mu_{1}$ as follows:

$$
P\left(n_{2}, \mu_{1}\right)=\frac{\left(\begin{array}{c}
N \\
n_{2}
\end{array}\right)\left(\mu_{0} / \mu_{2}\right)^{n_{2}}+N !\left(\mu_{0} / \mu_{1}\right)^{N} \sum_{n=0}^{N-n_{2}-1}\left(\mu_{1} / \mu_{0}\right)^{n+n_{2}} / n !}{\sum_{m=0}^{S}\left(\begin{array}{c}
N \\
m
\end{array}\right)\left(\mu_{0} / \mu_{2}\right)^{m}+N !\left(\mu_{0} / \mu_{1}\right)^{N} \sum_{m=0}^{S} \sum_{n=0}^{N-m-1}\left(\mu_{0} / \mu_{1}\right)^{n+m} / n !} .
$$

Clearly, as $\mu_{1}$ approaches infinity, $P\left(n_{2}, \mu_{1}\right)$ approaches the marginal distribution of an Engset loss station. One can further show that the time congestion at station 2 , given by $P\left(S, \mu_{1}\right)$, is a monotonically increasing function of $\mu_{1}$. The behavior of $P\left(S, \mu_{1}\right)$ as a function of $\mu_{1}$ quantifies the tradeoff between blocking at station 2 and queueing delay in station 1 . As $\mu_{1} \rightarrow \infty$, the queueing delay decreases to zero but the time congestion at station 2 increases to that of an Engset loss station. The effect of the queueing station is to alleviate blocking at the loss station at the expense of introducing queueing delay.

We now point out an equivalence between the QLN of Figure 7 and the open loss network (OLN) defined in Section 3. Using the fact that the variables in Eq.(29) must satisfy $n_{0}+n_{1}+$ $n_{2}=N$, we can write the stationary distribution of $\left(n_{0}, n_{2}\right)$ as:

$$
P\left(n_{0}, n_{2}\right) \propto \frac{1}{n_{0} !}\left(\frac{\mu_{1}}{\mu_{0}}\right)^{n_{0}} \frac{1}{n_{2} !}\left(\frac{\mu_{1}}{\mu_{2}}\right)^{n_{2}} .
$$

Hence,

$$
P\left(n_{0}, n_{2}\right)=\frac{1}{\tilde{G}(S, N)} \frac{a_{0}^{n_{0}}}{n_{0} !} \frac{a_{2}^{n_{2}}}{n_{2} !}
$$

where $a_{0}=\mu_{1} / \mu_{0}, a_{2}=\mu_{1} / \mu_{2}$ and

$$
\tilde{G}(S, N)=\sum_{0 \leq n_{0}+n_{2} \leq N, n_{2} \leq S} \frac{a_{0}^{n_{0}}}{n_{0} !} \frac{a_{2}^{n_{2}}}{n_{2} !} .
$$

Observe that Eq.(41) is the stationary state distribution of an open loss network defined as follows. There are two links, $l_{1}$ and $l_{2}$, with link capacities $N$ and $S$, respectively. There are two traffic classes, $c_{0}$ and $c_{2}$ : Calls of class $c_{0}$ arrive according to a Poisson process of rate $a_{0}$ and use a route containing just link $l_{1}$. Calls of class $c_{2}$ arrive according to a Poisson process of 
rate $a_{2}$ and use a route containing links $l_{1}$ and $l_{2}$. The matrix $A=\left[A_{l c}: l \in\left\{l_{1}, l_{2}\right\}, c \in\left\{c_{0}, c_{2}\right\}\right]$ which indicates the resource requirements of the OLN is given as follows:

$$
A=\left[\begin{array}{ll}
1 & 1 \\
0 & 1
\end{array}\right]
$$

Thus, with respect to the stationary probability state distribution of the two loss stations in Figure 7, the simple QLN is equivalent to an OLN. Furthermore, certain methods for approximate the normalization constant $\tilde{G}(S, N)$ for the OLN may be used to solve for the probability state distribution of the QLN.

\section{Conclusion}

In this paper we introduced the queueing-loss network model as a generalization of classical loss and queueing models. By further generalizing results on loss networks (Kobayashi \& Mark, 1994, 1997), we showed that the product-form solution applies to this extended class of stochastic models. Queueing-loss networks allow multiple classes of calls, multiple types of servers, general call service times and general call inter-generation time distributions. A key observation in making this generalization was that an entire open loss network (OLN) or subnetwork could be replaced by a single generalized loss station (GLS).

The queueing-loss network can be used to model systems which involve both queueing and loss behaviors. For example, arriving calls to a circuit-switched network may first have to wait at a queueing station prior to being subjected to admission control. For a small three-stage closed queueing-loss network, we have shown how various performance measures can be calculated. For general queueing-loss networks, performance measures such as time congestion, call congestion at loss stations, and utilization at queueing stations can be expressed in terms of the normalization constant $G(\mathbf{S}, \mathbf{N})$. For a large network with large values of $\mathbf{S}$ (the vector of number of servers at various loss stations) and/or large $\mathbf{N}$ (the vector of the number of sources in closed subchains), a direct evaluation of the normalization constants $G(\mathbf{S}, \mathbf{N})$ become computationally intensive. A large body of literature exists that addresses the computational aspect of approximating the normalization constant for loss networks (see e.g., (Kobayashi \& Mark, 1997) and references cited therein).

Future work could investigate the behaviors of more complicated queueing-loss network models and in particular, the interaction between the queueing and loss aspects. A limitation of the queueing-loss network models discussed in this paper is that the subsequent behavior of calls, after being blocked at a loss station, is identical with that of calls which have successfully received service; i.e., they proceed to follow the same path. In practice, blocked calls are often tagged as such and subsequently receive different treatment from their successfully served counterparts. Although the product-form solution will no longer hold in this case, the practical implications of such a model make its thorough investigation an interesting open problem.

\section{Acknowledgements}


This research has been supported, in part, by the National Science Foundation, the New Jersey Commission on Science and Technology, and the Ogasawara Foundation for the Promotion of Science and Technology.

\section{References}

Baskett, F., Chandy, K.M., Muntz R.R., and Palacios, F.C. (1975). Open, closed, and mixed networks of queues with different classes of customers. Journal ACM, Vol. 22, No. 2, pp. 248-260.

Cohen, J.W. (1957). The Generalized Engset Formulae. Philips Telecommunication Review, Vol. 18, No. 4, pp. 158-170.

Jackson, J.R. (1963). Jobshop-like Queueing Systems, Management Science, Vol. 10, No. 1, pp. 131-142 .

Kelly, F.P. (1991). Loss networks. Annals of Applied Probability, Vol. 1, No. 3, pp. 319-378.

Kelly, F.P. (1979). Reversibility and Stochastic Networks, Wiley, Chichester.

Kobayashi, H. (1978). Modeling and Analysis: An Introduction to System Performance Evaluation Methodology, Addison-Wesley.

Kobayashi, H \& Mark, B.L. (1994). On Queueing Networks and Loss Networks, Proc. of the 28th Annual Conference on Information Sciences and Systems, pp. 794-799, Princeton, New Jersey.

Kobayashi, H. \& Mark, B.L. (1997). Product-Form Loss Networks. In J. Dshalalow (Ed) Frontiers in Queueing: Models and Applications in Engineering and Science, pp. 147-195, CRC Press.

Reiser, M. \& Kobayashi, H. (1975). Queueing networks with multiple closed chains: theory and computational algorithms. IBM Journal of Research and Development, Vol. 19, pp. 283-294.

Syski, R. (1986). Introduction to Congestion Theory in Telephone Systems, Second Edition, Amsterdam: Elsevier Science Publisher. 1986.

Wolff, R. (1989). Stochastic Modeling and the Theory of Queues, Englewood Cliffs: PrenticeHall. 